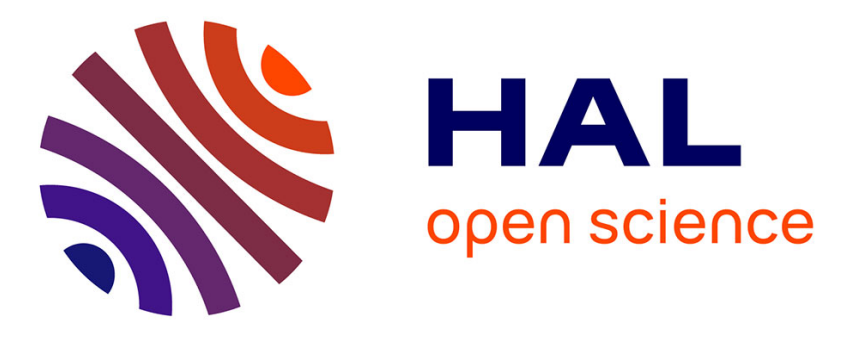

\title{
A dwarf disrupting - Andromeda XXVII and the North West Stream
}

\author{
Janet Preston, Michelle Collins, Rodrigo Ibata, Erik Tollerud, R Michael \\ Rich, Ana Bonaca, Alan Mcconnachie, Dougal Mackey, Geraint Lewis, Nicolas \\ F. Martin, et al.
}

\section{To cite this version:}

Janet Preston, Michelle Collins, Rodrigo Ibata, Erik Tollerud, R Michael Rich, et al.. A dwarf disrupting - Andromeda XXVII and the North West Stream. Monthly Notices of the Royal Astronomical Society, 2019, 490 (2), pp.2905-2917. 10.1093/mnras/stz2529 . hal-03095297

\section{HAL Id: hal-03095297 https://hal.science/hal-03095297}

Submitted on 17 Jan 2021

HAL is a multi-disciplinary open access archive for the deposit and dissemination of scientific research documents, whether they are published or not. The documents may come from teaching and research institutions in France or abroad, or from public or private research centers.
L'archive ouverte pluridisciplinaire HAL, est destinée au dépôt et à la diffusion de documents scientifiques de niveau recherche, publiés ou non, émanant des établissements d'enseignement et de recherche français ou étrangers, des laboratoires publics ou privés. 


\title{
A Dwarf Disrupting - Andromeda XXVII and the North West Stream
}

\author{
Janet Preston, ${ }^{1 *}$ Michelle L.M. Collins, ${ }^{1}$ Rodrigo A. Ibata, ${ }^{2}$ Erik J. Tollerud, ${ }^{3}$ \\ R. Michael Rich ${ }^{4}$ Ana Bonaca, ${ }^{5}$ Alan W. McConnachie ${ }^{6}$ Dougal Mackey $^{7}$ \\ Geraint F. Lewis ${ }^{8}$ Nicolas F. Martin ${ }^{2,9}$ Jorge Peñarrubia ${ }^{10}$ Scott C. Chapman ${ }^{11}$ \\ Maxime Delorme ${ }^{1}$ \\ ${ }^{1}$ Department of Physics, University of Surrey, Guildford, GU2 7XH, Surrey, UK. * \\ ${ }^{2}$ Observatoire de Strasbourg, 11, rue de l'Université, F-67000, Strasbourg \\ ${ }^{3}$ Space Telescope Science Institute, 3700 San Martin Drive, Baltimore, MD 21218, USA \\ ${ }^{4}$ Department of Physics and Astronomy, University of California at Los Angeles, Los Angeles, CA 90095, USA \\ ${ }^{5}$ Harvard-Smithsonian Center for Astrophysics, 60 Garden St, Cambridge, MA 02138, USA \\ ${ }^{6}$ NRC Herzberg Institute of Astrophysics, 5071 West Saanich Road, Victoria, B.C., V9E 2E7, Canada \\ ${ }^{7}$ Research School of Astronomy and Astrophysics, Australian National University, Canberra, ACT 2611, Australia \\ ${ }^{8}$ Sydney Institute for Astronomy, School of Physics A28, The University of Sydney, NSW, 2006, Australia \\ ${ }^{9}$ Max-Planck-Institut für Astronomie, Königstuhl 17, D-69117 Heidelberg, Germany \\ ${ }^{10}$ Institute for Astronomy, University of Edinburgh, Royal Observatory, Blackford Hill, Edinburgh EH9 3HJ, UK \\ ${ }^{11}$ Department of Physics and Atmospheric Science, Dalhousie University, 6310 Coburg Road, Halifax, B3H 4R2, Canada
}

Accepted XXX. Received YYY; in original form ZZZ

\begin{abstract}
We present a kinematic and spectroscopic analysis of 38 red giant branch stars, in 7 fields, spanning the dwarf spheroidal galaxy Andromeda XXVII and the upper segment of the North West Stream. Both features are located in the outer halo of the Andromeda galaxy at a projected radius of 50-80 kpc, with the stream extending for $\sim 3^{\circ}$ on the sky. Our data is obtained as part of the PAndAS survey and enables us to confirm that Andromeda XXVII's heliocentric distance is $827 \pm 47 \mathrm{kpc}$ and spectroscopic metallicity is $-2.1_{-0.5}^{+0.4}$. We also re-derive Andromeda XXVII's kinematic properties, measuring a systemic velocity $=-526.1_{-11.0}^{+10.0} \mathrm{kms}^{-1}$ and a velocity dispersion that we find to be non-Gaussian but for which we derive a formal value of $27.0_{-3.9}^{+2.2} \mathrm{kms}^{-1}$. In the upper segment of the North West Stream we measure mean values for the metallicity $=-1.8 \pm 0.4$, systemic velocity $=-519.4 \pm 4.0 \mathrm{kms}^{-1}$ and velocity dispersion $=10.0 \pm 4.0 \mathrm{kms}^{-1}$. We also detect a velocity gradient of $1.7 \pm 0.3 \mathrm{kms}^{-1} \mathrm{kpc}^{-1}$ on an infall trajectory towards M31. With a similar gradient, acting in the same direction, in the lower segment we suggest that the North West Stream is not a single structure. As the properties of the upper segment of the North West Stream and Andromeda XXVII are consistent within $90 \%$ confidence limits, it is likely that the two are related and plausible that Andromeda XXVII is the progenitor of this stream.
\end{abstract}

Key words: galaxies: dwarf - galaxies: fundamental parameters - galaxies: kinematics and dynamics - Local Group

\section{INTRODUCTION}

The spectacular stellar streams snaking around the Milky Way (MW) and Andromeda (M31) are the paleontological

^j.preston@surrey.ac.uk

(C) 2017 The Authors remnants of mergers on a galactic scale. In the past decade, wide and deep photometric surveys, such as the Dark Energy Survey (DES, Abbott et al. 2005, Shipp et al. 2018), the PanAndromeda Archaeological Survey (PAndAS, McConnachie et al. 2009), the Sloan Digital Sky Survey (SDSS, Ahn et al. 2014), the Pan-STARRS1 $3 \pi$ Survey (PS1, Bernard et al. 


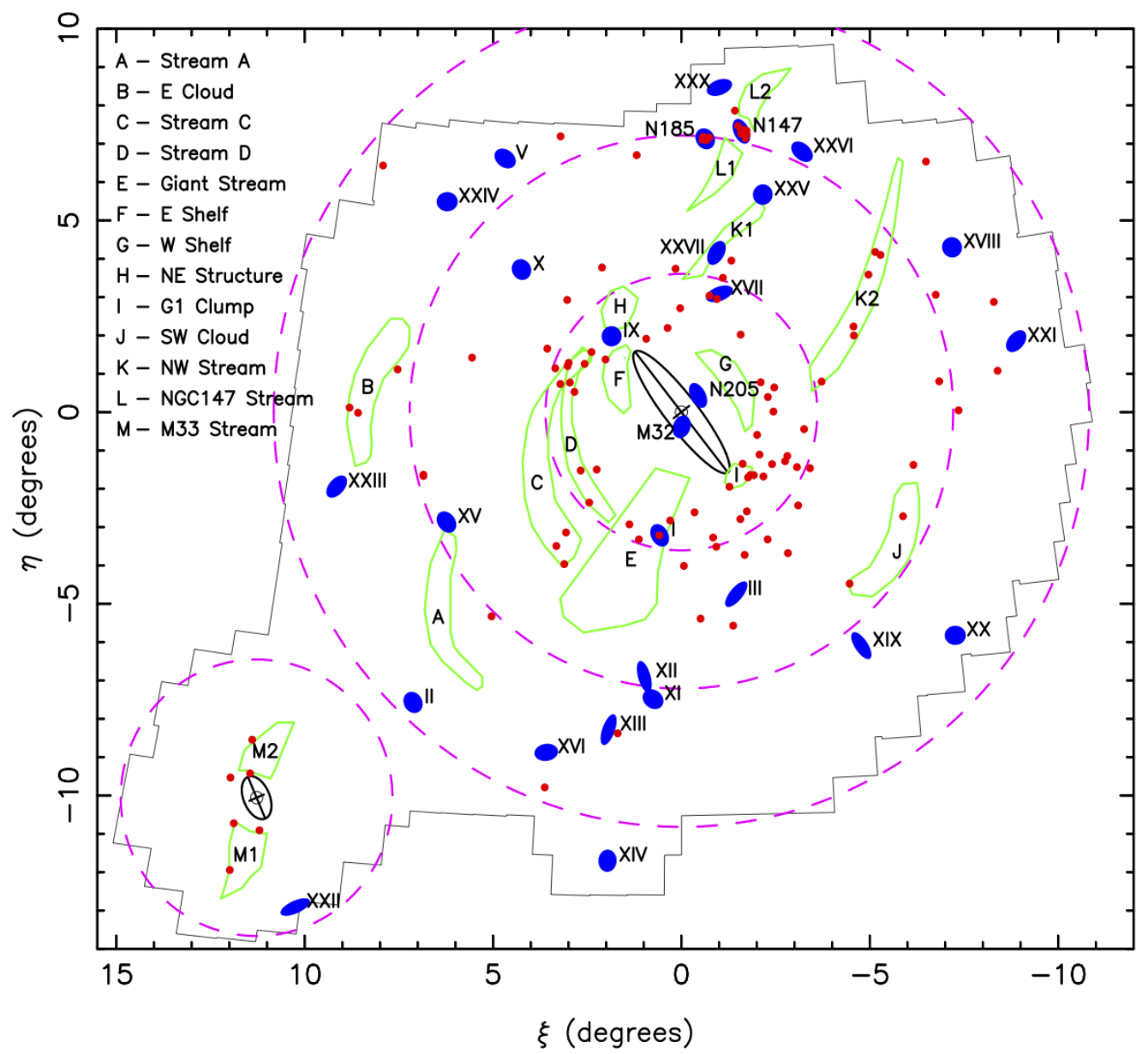

Figure 1: And XXVII and NW stream within the PAndAS footprint. The dashed circles show projected radii of 50kpc, 100kpc and $150 \mathrm{kpc}$ from M31, and 50kpc from M33. The grey polygon denotes the outline of the PAndAS footprint. Red dots indicate known globular clusters at projected radii of greater than 1 degree from M31. Blue ellipses correspond to known dwarf galaxies in the PAndAS footprint. Other stellar substructures in the halo of M31 are outlined in green. Figure reproduced from McConnachie et al. (2018).

2016) and the ESA/Gaia survey (The Gaia Collaboration 2016) have discovered more than 60 streams around the MW (Grillmair \& Carlin 2016, Shipp et al. 2018, Malhan \& Ibata 2018 and Ibata et al. 2019) and more than 10 streams around M31 (Martin et al. 2014a, Ibata et al. 2014, Ferguson \& Mackey 2016, McConnachie et al. 2018).

These streams comprise debris from the tidal disruption of smaller stellar structures, such as dwarf galaxies and globular clusters, as they orbit around their host galaxies. They provide visible evidence that large galaxies grow by assimilating smaller ones. Using data from stellar streams we can create models to show how small galaxies are accreted by larger ones, the timescales over which this happens and test the $\Lambda \mathrm{CDM}$ paradigm of hierarchical galaxy formation (Press \& Schechter 1974, Springel et al. 2006, Frenck \& White 2012). We also know that the stellar debris within a stream follows the orbital path of its progenitor. So, using Newton's law of attraction, we can constrain the gravitational potentials, masses and dark matter distributions of the host galaxies (Ibata et al. 2002, 2004, Chapman et al. 2006, Koposov et al. 2010, Carlberg 2012, Fardal et al. 2013, Lux et al. 2013, Ibata et al. 2014, Erkal et al. 2016).

In the MW, several streams are presumed to have dwarf galaxy progenitors, for example: the Sagittarius (Ibata et al. 1994, Johnston et al. 1995, Mateo 1998, Grillmair \& Carlin 2016), Orphan (Grillmair 2006, Belokurov et al. 2007, Erkal et al. 2018, Koposov et al. 2018), Cetus Polar (Newberg et al. 2009), PAndAS MW (Martin et al. 2014a) and Styx (Grillmair 2009, Carlin \& Sand 2018) streams.

In M31, stream fields are found near NGC147 (Richardson et al. 2011, Lewis et al. 2013) and NGC205 (McConnachie et al. 2004, Ferguson \& Mackey 2016). In the inner halo, the Giant Stellar Stream, (Ibata et al. 2001, McConnachie et al. 2003, Ibata et al. 2004, Guhathakurta et al. 2006, Gilbert et al. 2009) the North-Eastern Shelf, labelled the NE Structure in Figure 1, (Zucker et al. 2004, Lewis et al. 2004) and the Western Shelf (Ferguson et al. 2002, 2005, 2006 and Fardal et al. 2012) are thought to be 
the tidal debris, from multiple pericentric passages of the M31 centre, of a progenitor with an estimated stellar mass $\sim 1-5 \times 10^{9} \mathrm{M}_{\odot}$. In the outer halo, questions still surround the progenitor of the luminous South West Cloud (Bate et al. 2014, Ferguson \& Mackey 2016) while the progenitor of the North West (NW) Stream is thought to be Andromeda XXVII (And XXVII), Richardson et al. (2011), McConnachie (2012), Collins et al. (2013), Martin et al. (2016).

The NW Stream comprises two segments. The lower segment (labelled K2 in Figure 1 and hereafter referred to as NW-K2) was discovered by McConnachie et al. (2009) using PAndAS data obtained from the $3.6 \mathrm{~m}$ Canada-FranceHawaii Telescope (CFHT). They find it to be $\sim 6^{\circ}(\sim 80 \mathrm{kpc})$ long in projection at a projected radius of $\sim 50-120 \mathrm{kpc}$ from the centre of M31. The upper segment (labelled K1 in Figure 1 and hereafter referred to as NW-K1) was discovered two years later by Richardson et al. (2011). They report this segment to be almost $\sim 3^{\circ}$ long in projection at a projected radius $\sim 50-80 \mathrm{kpc}$ from the centre of M31. Despite the two segments being quite separate, see Figure 1, based on their morphology Richardson et al. (2011) consider them to be part of a single stellar structure entwined around M31. This view is supported by Ibata et al. (2014), who detected similar metallicities in both segments of the stream, and by Carlberg et al. (2011). Their work indicates that the stars in the stream are $\sim 10$ Gyrs old, that the stream is $\sim 5 \mathrm{kpc}$ wide and that it extends for a projected distance $\sim 200 \mathrm{kpc}$, making it one of the longest in the Local Group (c.f. the MW's Sagittarius Stream, which is detected at heliocentric distances of $37 \mathrm{kpc} \leq \mathrm{D}_{\odot} \leq 117 \mathrm{kpc}$, implying an estimated length $\sim 80$ kpc, Belokurov et al. (2014), and the Giant Stellar Stream, to the South East of M31, with an estimated length of $\sim 100$ kpc, Gilbert et al. (2009)). Carlberg et al. (2011) report that NW-K2 is almost complete while NW-K1 contains a number of clearly visible gaps that could have been induced by dark matter sub-halos. Recent modelling of NW-K2 by Kirihara et al. (2017) indicates that a progenitor would need to have a stellar mass $\sim 10^{6-8} \mathrm{M}_{\odot}$ and a minimum $\mathrm{r}_{h} \geq 30 \mathrm{pc}$, which is consistent with it being a dwarf galaxy, and that the dSph And XXVII could be the progenitor of the full stream.

And XXVII was discovered, contemporaneously with NW-K1, by Richardson et al. (2011). Kinematic analysis by Collins et al. (2013) led these authors to agree with Richardson et al. that And XXVII is not in dynamical equilibrium and is no longer a bound system, a view also supported by Martin et al. (2016) and Cusano et al. (2017).

To provide more insight into this complex and intriguing dSph galaxy and, potentially, its tidal stream, we present in this paper a study of the stellar populations of And XXVII and NW-K1 to determine if the two are associated. Using dynamical data for stars in these features we determine their systemic velocities, velocity dispersions and metallicities. We also show that And XXVII could be the progenitor of NWK1 and that NW-K1 may be a separate feature from NW$\mathrm{K} 2$.

The paper is structured as follows: Section 2 describes our observations and the data reduction process, Section 3 describes our data analysis approach and we present our conclusions in Section 4.

\begin{tabular}{ll}
\hline Right Ascension(J2000) & $00^{h}: 37^{m}: 27^{s} .1 \pm 0^{s} .5$ \\
${\text { Declination }(\mathrm{J} 2000)^{(a)}}^{(a)}$ & $+45^{o}: 23^{m} 13^{s} .0 \pm 10^{s}$ \\
Distance from M31 $^{(a)}$ & $86 \pm 48 \mathrm{kpc}$ \\
$\mathrm{M}_{v}{ }^{(a)}$ & $-7.9 \pm 0.5$ \\
$\mathrm{r}_{h}{ }^{(a)}$ & $455 \pm 80 \mathrm{pc}$ \\
$\mathrm{Fe} / \mathrm{H}^{(b)}$ & $-2.1 \pm 0.5$ \\
$\mathrm{M}_{r_{h}}{ }^{(b)}$ & $8.3_{-3.9}^{+2.8} \times 10^{7} \mathrm{M}_{\odot}$ \\
$\mathrm{M} / \mathrm{L}\left(\mathrm{r}<r_{h}\right)^{(b)}$ & $1391_{-1128}^{+1039} \mathrm{M}_{\odot} / \mathrm{L}_{\odot}$ \\
${\text { Systemic velocity } \mathrm{v}_{r}}^{(b)}$ & $-539.6_{-4.5}^{+4.7} \mathrm{kms}^{-1}$ \\
${\text { Velocity dispersion } \sigma_{v}}^{(b)}$ & $14.8_{-3.1}^{+4.3} \mathrm{kms}^{-1}$ \\
\hline
\end{tabular}

Table 1: Properties of AndXXVII as determined by (a) Richardson et al. (2011) and (b) Collins et al. (2013)

\section{OBSERVATIONS}

\subsection{Photometry - CFHT}

Data for the initial observations were obtained as part of the PAndAS survey. PAndAS used the $3.6 \mathrm{~m} \mathrm{CFHT} \mathrm{with}$ the MegaPrime/MegaCam camera, comprising 36, $2048 \mathrm{x}$ 4612 , CCDs with a pixel scale of $0.185 \%$ / pixel, delivering an almost 1 degree $^{2}$ field of view (McConnachie et al. 2009). To enable good colour discrimination of Red Giant Branch

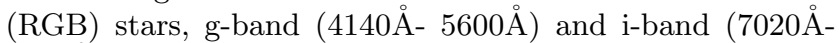
$8530 \AA)$ filters were used. Good seeing of $<0$ ". 8 enabled individual stars to be resolved to depths of $g=26.5$ and $i=$ 25.5 with a signal to noise ratio of $\sim 10$ (McConnachie et al. 2009, Collins et al. 2013, Martin et al. 2014b).

The data were initially processed by the Elixir system, Magnier \& Cuillandre (2004), at CFHT, which, in addition to ascertaining the photometric zero points, also de-biased, flat-fielded and fringe-corrected it. The data were then transferred to the Cambridge Astronomical Survey Unit to be further reduced using the bespoke pipeline described by Irwin \& Lewis (2001). Following this, the data were classified morphologically as, e.g., point source, non-point source and noise-like, then stored with band-merged $g$ and $i$ data (see Richardson et al. 2011). For this work we select point source objects.

\subsection{Spectroscopic Observation - Keck DEIMOS}

Data are obtained from seven masks (the on-sky positions of which are indicated in Figure 2, and the data from which are provided in the on-line version of this paper at Appendix A) spanning a distance of $\sim 120 \operatorname{arcmins}(\sim 30 \mathrm{kpc})$ of NWK1 and crossing the centre of And XXVII. Three of the masks were observed in 2010 and 2011 (603HaS, 604HaS and 7And27) and may have been analysed by Collins et al. (2013) and Martin et al. (2016), though they are not explicitly named in either work. The remaining four masks, A27sf1, A27sf2, A27sf3 and A27sf4, provide us with additional spectroscopic data obtained using the DEep-Imaging Multi-Object Spectrograph (DEIMOS) on the Keck II Telescope on the dates shown in Table 2. Our observations use the OG550 filter with the 1200 lines/mm grating with a resolution of $\sim 1.1 \AA-1.6 \AA$ at FWHM. Each mask/site is observed for 1 hour, split into 3 x 20 minute integrations. Observations 


\begin{tabular}{|c|c|c|c|c|c|c|c|c|}
\hline \multirow{2}{*}{ Mask name } & \multirow{2}{*}{ Date } & \multirow{2}{*}{ PI } & \multirow{2}{*}{$\alpha_{\mathrm{J} 2000}$} & \multirow{2}{*}{$\delta_{\mathrm{J} 2000}$} & \multirow{2}{*}{$\begin{array}{l}D_{\mathrm{A} 27} \\
\mathrm{kpc}\end{array}$} & \multicolumn{3}{|c|}{ No. of candidate stars within... } \\
\hline & & & & & & And XXVII & M31 & MW \\
\hline A27sf1 & 2015-09-12 & Collins & $00: 39: 39.96$ & $+45: 08: 47.73$ & 6.6 & 8 & 5 & 57 \\
\hline $603 \mathrm{HaS}$ & 2010-09-09 & Rich & $00: 38: 58.52$ & $+45: 17: 32.20$ & 4.1 & 8 & 4 & 53 \\
\hline 7And27 & $2011-09-26$ & Rich & $00: 37: 29.40$ & $+45: 24: 12.50$ & 0.3 & 11 & 4 & 54 \\
\hline A27sf2 & 2015-09-12 & Collins & $00: 36: 13.17$ & $+45: 32: 31.68$ & 3.8 & 2 & 8 & 49 \\
\hline $\mathrm{A} 27 \mathrm{sf} 4$ & 2015-09-12 & Collins & $00: 33: 28.25$ & $+45: 49: 24.87$ & 11.7 & 0 & 4 & 63 \\
\hline $604 \mathrm{HaS}$ & 2010-09-09 & Rich & 00:32:05.16 & $+46: 08: 31.20$ & 17.4 & 1 & 3 & 68 \\
\hline A27sf3 & 2015-09-12 & Collins & $00: 30: 25.60$ & $+46: 14: 52.66$ & 21.6 & 4 & 1 & 76 \\
\hline
\end{tabular}

Table 2: Properties for each observing mask, including: mask name; date observations were made; observing PI; Right Ascension and Declination of the centre of each mask; projected distance of the centre of the mask from And XXVII ( $\left.D_{\mathrm{A} 27}\right)$ and the number of stars likely to belong to each of the stellar populations (i.e. And XXVII, M31 and the MW) based on probability of membership. The $\alpha$ and $\delta$ for the centre of each mask are determined by taking the mean of the coordinates for all stars on the mask. The masks are listed in order of increasing distance from M31.
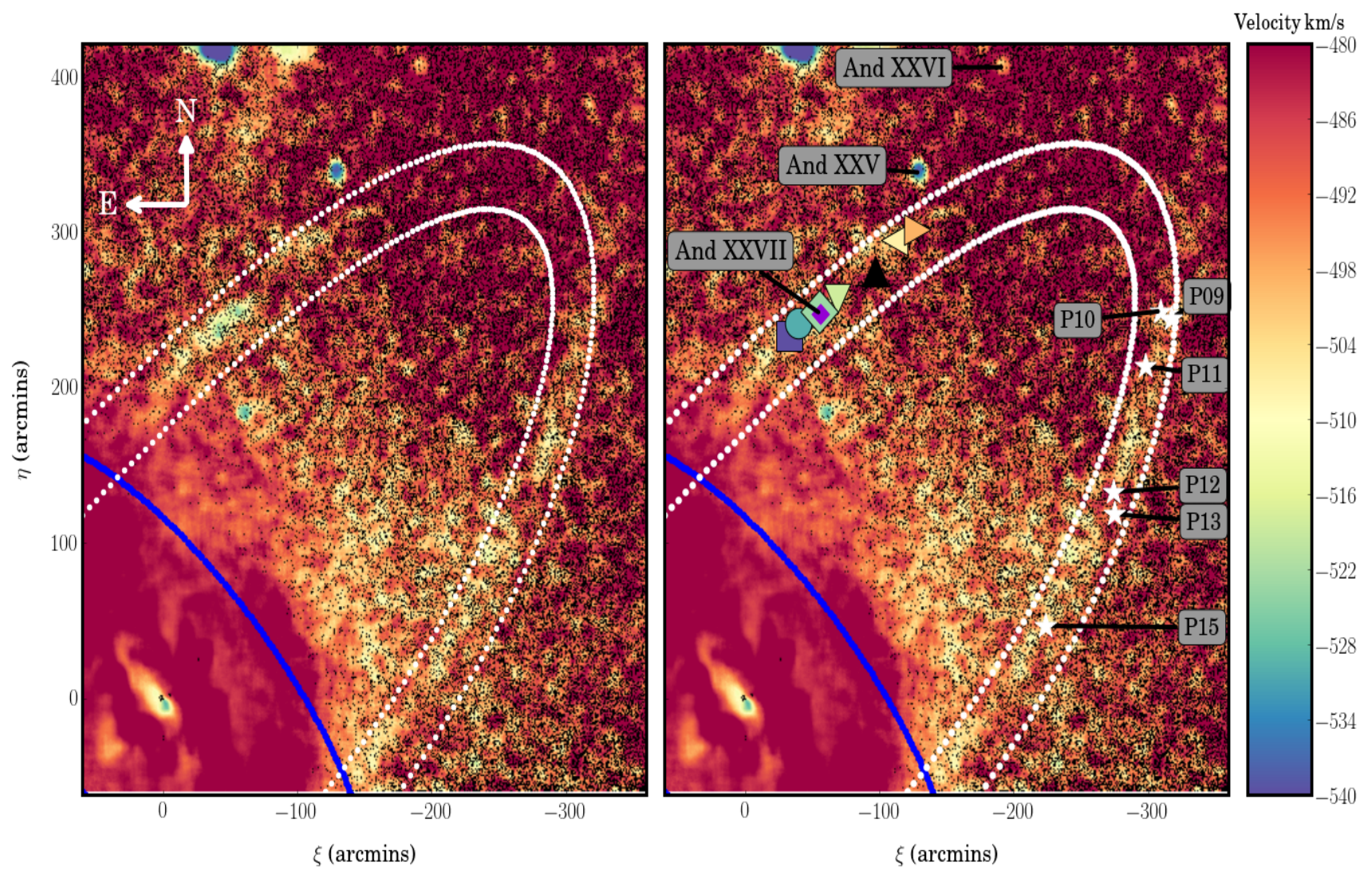

Figure 2: Density plot of RGB stars in the area around And XXVII and the NW stream. The data are obtained from the PAndAS catalogue for stars with $20.5 \leq i_{0} \leq 24.5$ and $-2.0 \leq[\mathrm{Fe} / \mathrm{H}] \leq-0.5$ and plotted in tangent plane standard coordinates centred on M31. The solid blue line represents the M31 halo (taking a semi-major axis of $55 \mathrm{kpc}$ with a flattening of 0.6, Ferguson \& Mackey 2016). The left-hand panel includes dotted, white, lines outlining the inner and outer edges of an ellipse tracing the possible track of the NW Stream, assuming it to be a single feature (following the approach by Carlberg et al. 2011). The right-hand panel shows the same data overlaid with the on-sky positions of the observing masks, which are represented by: square $=603 \mathrm{HaS} ;$ circle $=\mathrm{A} 27 \mathrm{sf1}$; diamond $=7 \mathrm{And}$ 27 (inset with a smaller, purple, diamond indicating the centre of And XXVII); inverted triangle = A27sf2; black triangle = A27sf4; left-pointing triangle $=604 \mathrm{HaS}$ and right-pointing triangle $=\mathrm{A} 27 \mathrm{sf} 3$. The icons are coloured coded by the systemic velocities derived later in this work, except for A27sf4, for which no And XXVII/NW-K1 candidates were found and so is coloured black. The Figure also indicates the relative positions of two other M31 satellites, And XXV and And XXVI (Richardson et al. 2011) and globular clusters, PAndAS-09 (P09) - PAndAS-15 (P15) in NW-K2 (Veljanoski et al. 2014). 
focus on the Calcium Triplet (CaT) region located between wavelengths $8400 \AA$ and $8700 \AA$.

We prioritise our targets by selecting stars based on their location within the colour magnitude diagram (CMD). Our highest priority targets are bright stars which lie directly on the And XXVII RGB with $20.3<i_{0}<22.5$. Our next priority are fainter stars on the RGB, i.e. $22.5<i_{0}<$ 23.5. We then fill the remainder of the mask with stars with $20.5<i_{0}<23.5$ and $0.0<g$ - $i<4.0$.

The data are reduced using a specifically constructed pipeline, described in Ibata et al. (2011), that corrects for: scattered light, flat-fields, the slit function and illumination within the telescope and calibrates the wavelength of each pixel. The final phase of the pipeline determines the velocities and associated uncertainties for the stars by: (1) creating model spectra comprising a continuum and the absorption profiles of the CaT lines (at $8498 \AA, 8542 \AA$ and $8662 \AA$ ); (2) cross-correlating these models with non-resampled stellar spectra using a Markov Chain Monte Carlo (MCMC) approach to obtain the optimum Doppler shift and CaT line widths; and (3) correcting the velocities and associated uncertainties, obtained from the posteriors of the above analysis, to the heliocentric frame.

\section{ANALYSIS OF AND XXVII AND NW-K1}

To enable us to determine if And XXVII and NW-K1 are associated we identify and confirm members of their stellar populations, determine the systemic velocities and velocity dispersions of these populations and obtain their metallicities.

\subsection{Stellar Populations}

To avoid any obvious failures of the pipeline and to remove velocities with high uncertainties, we select stars that have velocities in the range $-650 \mathrm{kms}^{-1}$ to $50 \mathrm{kms}^{-1}$ with velocity uncertainties $<20 \mathrm{kms}^{-1}$. Applying these criteria we find no And XXVII/NW-K1 candidate stars on mask A27sf4, which leaves us with a potential gap in the stream. Since this location is targeted on the same basis as the other masks, we conclude that the stream, at this point, must have a lower density than the surrounding areas. This is consistent with Carlberg et al. (2011) who found significant density variations along the NW-K1 stream.

We plot velocity histograms for the remaining masks, see Figure 3. These reveal three kinematically distinct stellar populations: stars likely to be members of the MW $\left(\mathrm{v}_{r}\right.$ $=\sim-80 \mathrm{kms}^{-1}$, Collins et al. 2013), stars likely to be members of the M31 halo (systemic velocity $\sim-300 \mathrm{kms}^{-1}$, Ibata et al. 2005b) and stars consistent with previously published values for the systemic velocity of And XXVII, $\mathrm{v}_{r},=-$ 539.6 ${ }_{-4.5}^{+4.7} \mathrm{kms}^{-1}$ (Collins et al. 2013).

To confirm if the stars in this latter category are members of the And XXVII/NW-K1 population we first look at their proximity to a fiducial isochrone. Richardson et al. (2011) find the age of And XXVII to be 12 Gyr and its metallicity $[\mathrm{Fe} / \mathrm{H}] \sim-1.7$, so we select an isochrone with these properties and $[\alpha / \mathrm{H}]=0.0$ from the Dartmouth Stellar Evolution database, Dotter et al. (2008). We correct for extinction and the heliocentric distance of And XXVII (827 kpc) and overlay it on the And XXVII RGB. We use the isochrone solely as a fiducial ridge-line for the And XXVII RGB. It is not used to derive or affect any properties reported in this paper, other than a given star's proximity to the RGB. Potential members of And XXVII and NW-K1 should fall along this isochrone, with stars lying close to it more likely to be members than those further away. Following the technique described by Tollerud et al. (2012), we assign a probability of membership to each star based on its proximity to the isochrone using:

$P_{\text {iso }}=\exp \left(\frac{-\Delta(\mathrm{g}-\mathrm{i})^{2}}{2 \sigma_{\mathrm{c}}}-\frac{\Delta(\mathrm{i})^{2}}{2 \sigma_{\mathrm{m}}}\right)$

where $\Delta(g-i)$ and $\Delta(i)$ are distances from the isochrone and $\sigma_{c}$ (which takes into account the range of colours of the stars on the CMD) and $\sigma_{m}$ (which factors in distance and photometric errors) are free parameters. Tollerud et al.'s values were adopted as the starting point and adjusted until values were obtained where stars that lie far from the And XXVII RGB have a low probability of association with the isochrone. We find the optimum values to be: $\sigma_{c}=0.15$ and $\sigma_{m}=0.45$.

Based on their location in the CMD, see Figure 4, we reject two stars from our candidate And XXVII/NW-K1 populations due to their lack of proximity to the isochrone. These are star number 80 on mask A27sf1 and star number 65 on mask $604 \mathrm{HaS}$.

To further refine our selection of candidate And XXVII/NW-K1 stars, we return to their velocities. To determine which stellar population a given star of velocity, $v_{i}$ and a velocity uncertainty of $v_{\text {err, }}$ is most likely to belong to, we define a single Gaussian function for each of them of the form:

$$
\begin{aligned}
P_{\text {struc }}= & \frac{1}{\sqrt{2 \pi\left(\sigma_{v, \text { struc }}^{2}+v_{\mathrm{err}, i}^{2}+\sigma_{\mathrm{sys}}^{2}\right)}} \times \\
& \exp \left[-\frac{1}{2}\left(\frac{v_{r, \text { struc }}-v_{r, i}}{\sqrt{\left.\sigma_{v, \text { struc }}^{2}+v_{\mathrm{err}, i}^{2}+\sigma_{\mathrm{sys}}^{2}\right)}}\right)^{2}\right]
\end{aligned}
$$

where: $P_{\text {struc }}$ is the resulting probability distribution function (pdf); $v_{\text {struc }} \mathrm{kms}^{-1}$ is the systemic velocity; $\sigma_{v \text {, struc }} \mathrm{kms}^{-1}$ is the velocity dispersion and $\sigma_{\text {sys }}$ is a systematic uncertainty component of $2.2 \mathrm{kms}^{-1}$, determined by Simon \& Geha (2007), Kalirai et al. (2010) and Tollerud et al. (2012) $)^{1}$, and contemporary with our observations. The likelihood function for membership of And XXVII, based on velocity, is then defined as:

$$
\begin{aligned}
\log \left[\mathcal{L}\left(v_{r}, \sigma_{r}\right)\right]=\sum_{i=1}^{N} \log \left(\eta_{M 31} P_{i, M 31}+\right. \\
\left.\eta_{M W} P_{i, M W}+\eta_{A 27} P_{i, A 27}\right)
\end{aligned}
$$

1 In calculating the uncertainties for the radial velocity measurements and telluric corrections of stars in their datasets, these authors find that an additional term, $\sigma_{\text {sys }}$, is required to obtain an accurate Gaussian distribution for their data. Repeated measurements of independent observations determine this to be $2.2 \mathrm{kms}^{-1}$ to take into account systematics not included in their Monte Carlo analyses. 

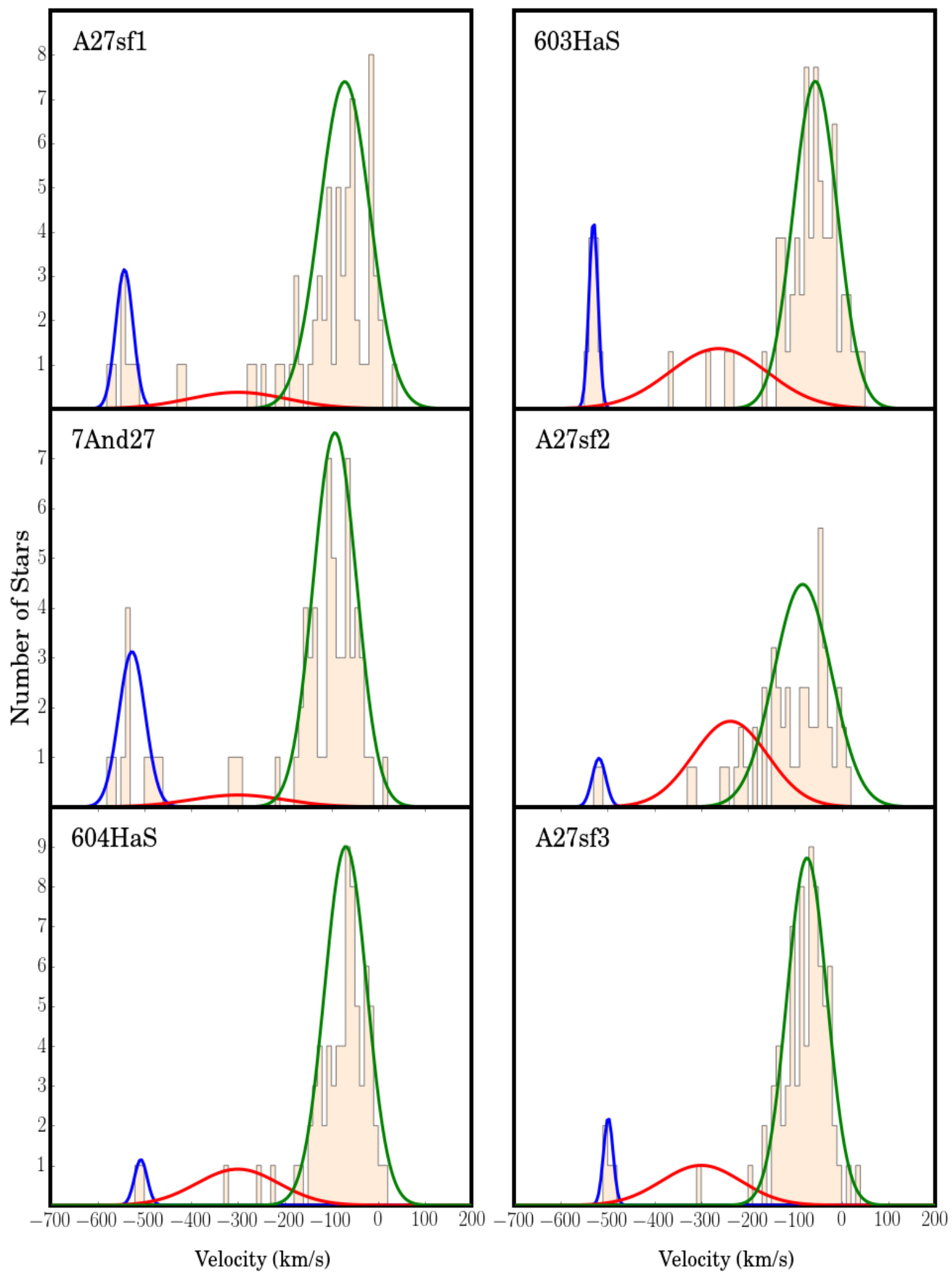

Figure 3: Kinematic analysis of And XXVII and NW-K1 showing velocity histograms overlaid with the membership pdf for each of the three stellar populations - coloured blue for And XXVII/NW-K1, red for M31 and green for the MW. 


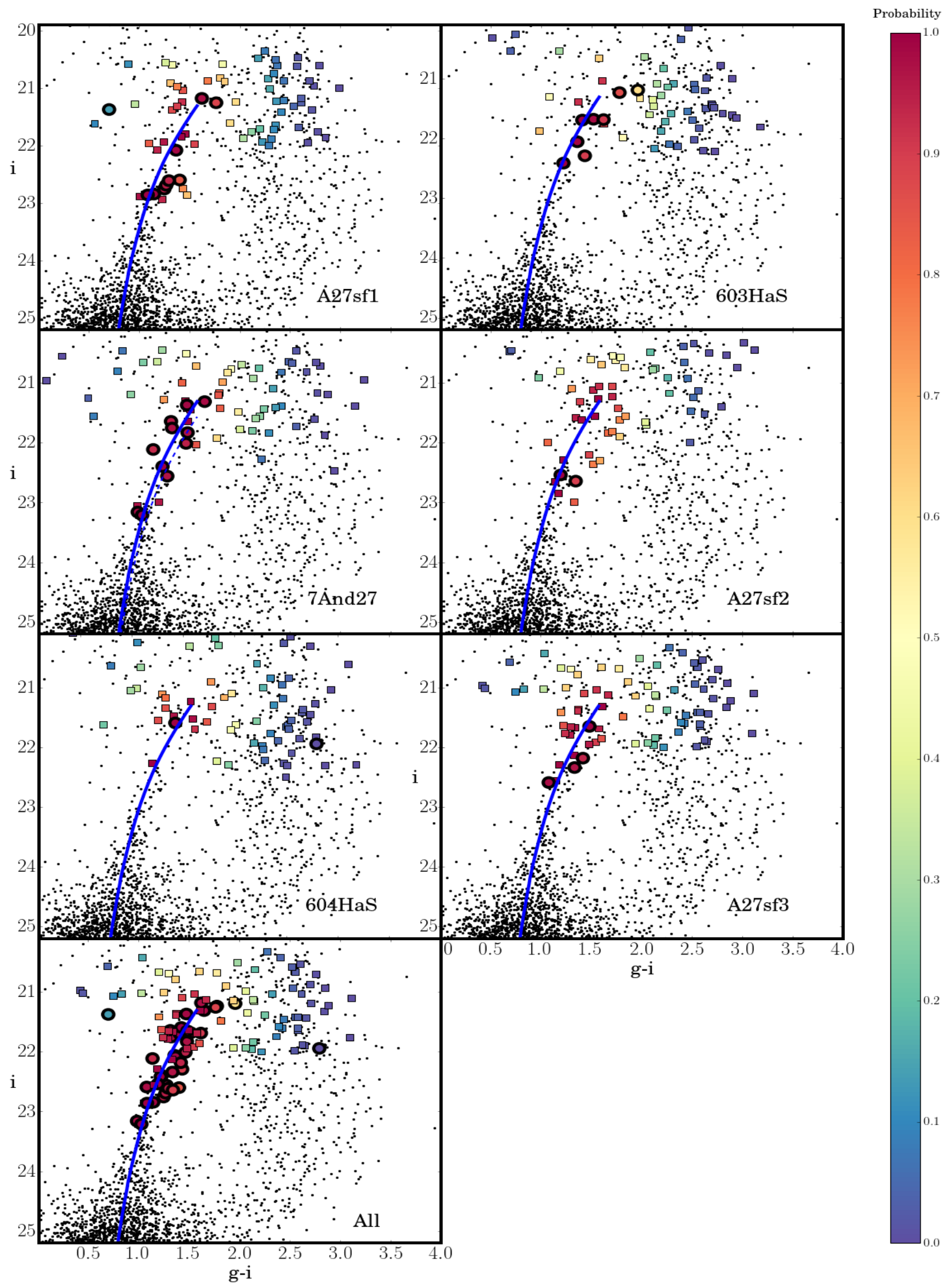

Figure 4: CMD for And XXVII/NW-K1 masks with a $12 \mathrm{Gyr},[\mathrm{Fe} / \mathrm{H}]=-1.7,[\alpha / \mathrm{H}]=0.0$ isochrone, corrected for extinction and an heliocentric distance of $827 \mathrm{kpc}$. The small black dots show stars from the PAndAS catalogue that lie within 5 arcmins of the centre of the respective masks. The dashed line in the plot for $7 \mathrm{And} 27$ shows the position of the same isochrone distance corrected to $1255 \mathrm{kpc}$ (a previously reported heliocentric distance for And XXVII). Round icons represent candidate And XXVII/NW-K1 stars. Square icons represent candidate MW and M31 halo stars. These first cut, candidate populations are based on stellar velocities. The icons are colour coded by probability of membership of And XXVII and NW-K1 based on proximity to the isochrone. The bottom right panel shows the combined results for all masks. 
where $\eta_{M 31}, \eta_{M W}$ and $\eta_{A 27}$ are the fraction of stars within each stellar population, $\mathrm{v}_{r}$ includes $\mathrm{v}_{r \text { A27 }}, \mathrm{v}_{r}$ M31 and $\mathrm{v}_{r} \mathrm{MW}$ and $\sigma_{r}$ includes $\sigma_{r \mathrm{~A} 27}, \sigma_{r \mathrm{M} 31}$ and $\sigma_{r \mathrm{MW}}$.

We incorporate the above equations, tailored for each stellar population (i.e. MW, M31, And XXVII/NW-K1) into an MCMC analysis, using the EMCEE software algorithm, Goodman \& Weare (2010), Foreman-Mackey et al. (2013). We set the initial values of the systemic velocity to previously published values, with the initial values for the velocity dispersion for And XXVII and the MW based on the spread of velocities in respective stellar populations. For M31 we calculate the initial value for the velocity dispersion in accordance with the the distance, $R$, of the centre of the mask from the centre of M31 (see Chapman et al. 2006 and Mackey et al. 2013) given by:

$\sigma_{v}(R)=\left(152-0.9 \frac{R}{1 \mathrm{kpc}}\right) \mathrm{kms}^{-1} \mathrm{kpc}^{-1}$

We base the initial values of the fraction parameters $\eta_{M 31}$, $\eta_{M W}$ and $\eta_{A 27}$ on the distribution of stars plotted on velocity histograms for each mask.

We set broad priors for each stellar populations on the masks i.e. :

- systemic velocities are $-580 \leq v_{\mathrm{A} 27} / \mathrm{kms}^{-1} \leq-450,-400$ $\leq v_{\mathrm{M} 31} / \mathrm{kms}^{-1} \leq-200$ and $-150 \leq v_{\mathrm{MW}} / \mathrm{kms}^{-1} \leq-10$.

- velocity dispersions are $0 \leq \sigma_{v \mathrm{~A} 27} / \mathrm{kms}^{-1} \leq 30,0 \leq$ $\sigma_{v \mathrm{M} 31} / \mathrm{kms}^{-1} \leq 100$ (except for masks A27sf1 and $603 \mathrm{HaS}$, where the prior is defined as $\left.0 \leq \sigma_{v \mathrm{M} 31} / \mathrm{kms}^{-1} \leq 200\right)$; and $0 \leq \sigma_{v \mathrm{MW}} / \mathrm{kms}^{-1} \leq 150$.

- the fraction parameters are $0 \leq \eta \leq 1$ with $\eta_{\mathrm{A} 27}+\eta_{\mathrm{M} 31}$ $+\eta_{\mathrm{MW}}=1$.

As part of our analysis, we test the use of broader priors for $\sigma_{v \mathrm{~A} 27}$ before adopting those reported above. At values $>30 \mathrm{kms}^{-1}$ we find the resulting pdfs to be a poor fit for our sparse data and inclusive of M31 halo stars. We also note an increase in the acceptance fraction which implies a reduction in the quality of the results. So we adopt the range specified, with the upper limit of $30 \mathrm{kms}^{-1}$ more than twice the expected velocity dispersion for a dwarf galaxy.

We set our Bayesian analysis to run for 100 walkers taking 100000 steps, a burn-in of 1000 and a scale parameter set to the default value of 2 . We use the EMCEE algorithm to fit Gaussians and derive posterior distributions for the systemic velocity, velocity dispersion and the fraction parameters for our stellar populations.

We find that, for all masks, the MW population can be approximated by a single Gaussian of the above form (Equation 3.2) as can the stellar populations for And XXVII and NW-K1. With respect to M31, we find that for masks 7And27, A27sf1, A27sf3 and 604Has there are too few stars to constrain the M31 halo adequately, so we fix the systemic velocity of M31 (at $-300 \mathrm{kms}^{-1}$ ), the velocity dispersion (obtained using Equation 3.4) and the $\eta_{M 31}$ parameter. For masks $603 \mathrm{HaS}$ and A27sf2 we allow the EMCEE algorithm to fit all the data. The M31 halo is then constrained, or fixed, by a single Gaussian, again of the form shown in Equation 3.2 .

We check the Acceptance Fraction of our model to ensure that we have an appropriate number of independent samples to represent the data. We find, for all models, an

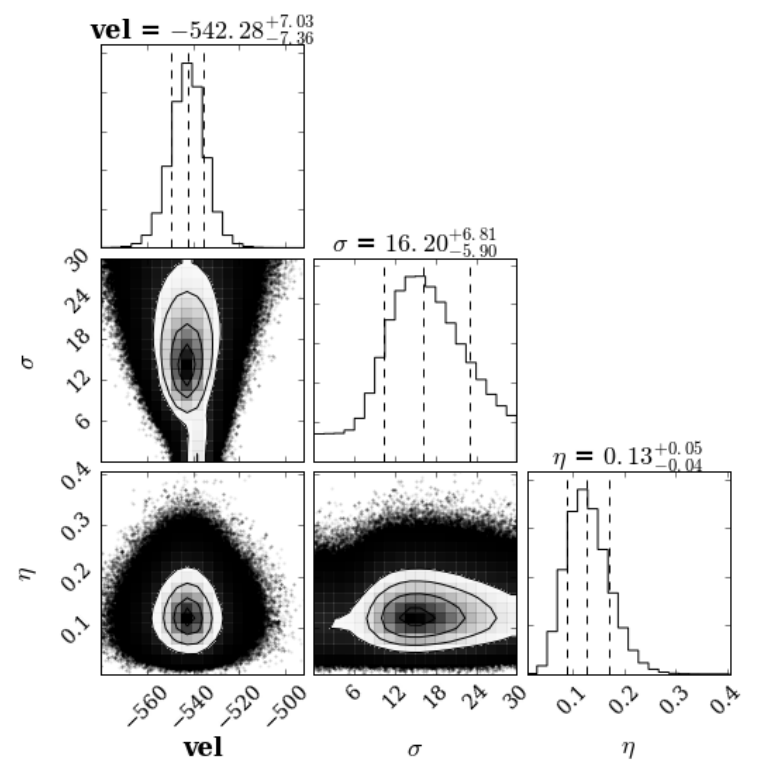

Figure 5: 2-d marginalised probability distribution functions for mask A27sf1 for systemic velocities (vel), velocity distributions $(\sigma)$ and percentage of stars in the stellar population $(\boldsymbol{\eta})$ for And XXVII. The dashed lines indicate the median values for each parameter and their 1- $\sigma$ uncertainties. This corner plot is created using code from Foreman-Mackey et al. (2016).

Acceptance Fraction $\sim 0.3$, which is in the range $(0.2-0.5)$ recommended by Hogg \& Foreman-Mackey (2017).

We also check that our MCMC chains are converged using the Integrated Autocorrelation Factor, obtained via the "acor" function in EMCEE. For each mask, we determine the precision, $\mathrm{p}$, of the values obtained for each parameter being estimated using: $\mathrm{p}=\sqrt{\tau / N}$, where $\tau$ is the autocorrelation time and $\mathrm{N}$ is the number of samples. We compare the values obtained $(\sim 0.003$ for each mask) with the uncertainties calculated by EMCEE for each parameter. As the precision is very much smaller than the uncertainties, we are satisfied that the chains are converged. We then use corner plots, such as the example shown at Figure 5, to visualise the distribution and covariance of the parameters.

Having fitted a Gaussian velocity profile for each of the three stellar populations, we derive the probabilities for each star on the masks belonging to a given population using:

$P_{\mathrm{vel}}=\frac{P_{A 27}}{P_{M 31}+P_{M W}+P_{A 27}}$

with the probability of being a contaminant given by:

$P_{\text {contam }}=\frac{P_{M 31}+P_{M W}}{P_{M 31}+P_{M W}+P_{A 27}}$

Figure 3 shows the velocity distribution histograms overlaid with the pdfs. In each we see a distinct cold peak located in the velocity range of $-650 \leq \mathrm{v} / \mathrm{kms}^{-1} \leq-400$. Based on these results, we reject one further star, number 16 on mask A27sf1, which has a low probability of association with And XXVII.

We assess the total probability of stars being members of And XXVII/NW-K1 by combining their probabilities of membership from the CMD and velocity analyses as follows: 


\begin{tabular}{lcc}
\hline Mask & $\begin{array}{c}\mathrm{v}_{r} \\
\mathrm{kms}^{-1}\end{array}$ & $\begin{array}{c}\sigma_{v} \\
\mathrm{kms}^{-1}\end{array}$ \\
\hline $\begin{array}{l}\text { And XXVII } \\
\text { 7And27(a) }\end{array}$ & $-526.1_{-11.0}^{+10.0}$ & $27.0_{-3.9}^{+2.2}$ \\
7And27(b) & $-536.5_{-2.3}^{+3.3}$ & $5.6_{-4.0}^{+6.1}$ \\
\hline NW-K1 & & \\
A27sf1 & $-542.3_{-7.4}^{+7.1}$ & $16.2_{-5.9}^{+6.9}$ \\
$603 \mathrm{HaS}$ & $-530.2_{-3.1}^{+2.9}$ & $5.1_{-3.3}^{+4.6}$ \\
A27sf2 & $-518.4_{-12.5}^{+12.5}$ & $11.7_{-8.3}^{+11.4}$ \\
604HaS & $-507.4_{-10.8}^{+10.7}$ & $10.9_{-7.8}^{+11.5}$ \\
A27sf3 & $-498.6_{-5.3}^{+5.3}$ & $6.2_{-4.4}^{+8.6}$ \\
Mean & $-519.4 \pm 4.0$ & $10.0^{4} 4.0$ \\
\hline
\end{tabular}

Table 3: Results of the kinematic analysis of And XXVII and NW-K1. For mask 7And27, values are shown for $(a)$ the single Gaussian fit to the And XXVII candidate stars and $(b)$ the cold peak. Values obtained from the posteriors of the EMCEE algorithm, with uncertainties reported at $68 \%$ confidence limits.

$P_{\mathrm{A} 27}=P_{\mathrm{vel}} \times P_{\text {iso }}$

We identify stars with $\mathrm{P}_{\mathrm{A} 27}>0.6$ as members of the stellar populations for And XXVII and NW-K1. We summarise the results of our kinematic analysis in Table 3 .

\subsubsection{Mask 7And27}

On review of our kinematic analysis of mask 7And27, located across the centre of And XXVII, we see that the single Gaussian is a poor fit for the data (see Figure 3). It does not adequately describe the clear cold peak and surrounding hotter components, which could be And XXVII or M31 halo stars. We also see that while the systemic velocity is inkeeping with the values obtained for the surrounding masks, the velocity dispersion is not (Figure 6, datapoint 7And27a).

We decide to fit two Gaussians to determine whether we could more accurately represent the data and address the above points. We set broad boundary priors for the hot components and tightly restricted priors to isolate the cold peak. Both Gaussians are given the same initial systemic velocity $\left(-536.0 \mathrm{kms}^{-1}\right)$ but different velocity dispersions of 5 $\mathrm{kms}^{-1}$ for the cold and $10 \mathrm{kms}^{-1}$ for the broad peak. Figure 7 shows the results of this approach. We find the cold peak well constrained with $\mathrm{v}_{r}=-536.5_{-2.3}^{+3.3} \mathrm{kms}^{-1}$ and a velocity dispersion, $\sigma_{v}=5.6_{-4.0}^{+6.1}$, which is more consistent with the neighbouring fields along the stream (Figure 6, datapoint 7And27b).

We note that neither model provides a really good fit for the data so we explore their relative quality using the extended Akaike information criterion, $\mathrm{AIC}_{c}$ (for use with small datasets) and the Bayesian information criterion, BIC, Akaike (1970), Burnham \& Anderson (2004). We calculate

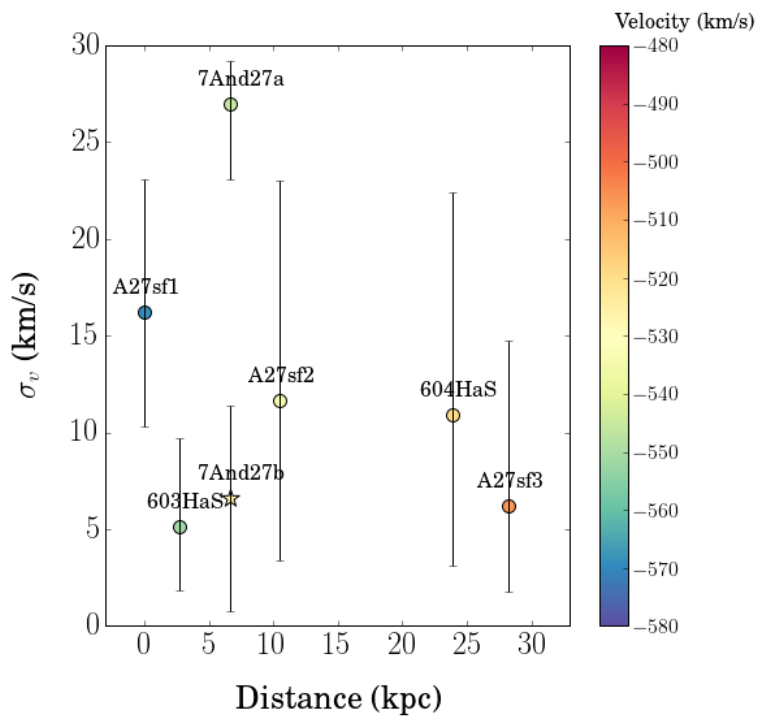

Figure 6: Velocity dispersions for all masks with respect to the centre of mask A27sf1 (which lies closest to M31). The values for the velocities and the error bars were obtained from the EMCEE algorithm, the mask locations were obtained using the mean value of all the coordinates for the stars on each respective mask. The datapoint labelled 7And27a represents the velocity dispersion obtained by fitting a single Gaussian to the data, while $7 \mathrm{And} 27 \mathrm{~b}$ is obtained by isolating the cold peak.

the values for these estimators using:

$A I C_{c}=-2 \log (\mathcal{L})+2 K+\frac{2 K(K+1)}{n-K-1}$

and:

$B I C=-2 \ln (\mathcal{L})+K$

where: $\mathcal{L}$ is the maximum value of the likelihood function for a given model, $\mathrm{K}$ is the number of parameters to be estimated and $\mathrm{n}$ is the number of data points in the analysis (in our case, the number of stars on the mask). Neither of these two estimators give any measure of the absolute quality or otherwise of any given model. Rather they provide insight into the quality of a given model relative to others. Both estimators encourage simplicity and will naturally favour the model with the fewest parameters to be fitted, so a model with a lower $\mathrm{AIC}_{c}$ or $\mathrm{BIC}$ value is deemed to be the "better" model. We find that the $\mathrm{AIC}_{c}$ and the $\mathrm{BIC}$ values for both models are of the same order of magnitude, with the single Gaussian model having a marginally $(\sim 3 \%)$ lower score for both. We, therefore, adopt the values from the single Gaussian model for $\mathrm{v}=-526.1_{-11.0}^{+10.0} \mathrm{kms}^{-1}$ and $\sigma_{v}=$ $27.0_{-3.9}^{+2.2} \mathrm{kms}^{-1}$ as the kinematic properties of And XXVII.

When we compare this to previous works we see that the systemic velocity is $\sim 13 \mathrm{kms}^{-1}$ lower and $\sigma_{v} \sim 13 \mathrm{kms}^{-1}$ higher than those reported by Collins et al. (2013). This is likely due to our analysis taking fields $603 \mathrm{HaS}$ and $7 \mathrm{And} 27$ separately rather than combining their data. Previously thought to lie at the centre of And XXVII, these two fields are $\sim 4 \mathrm{kpc}$ apart on the sky, which is a much larger distance than the $\mathrm{r}_{h}$ for And XXVII (455 pc). As the $\alpha$ and $\delta$ for the 


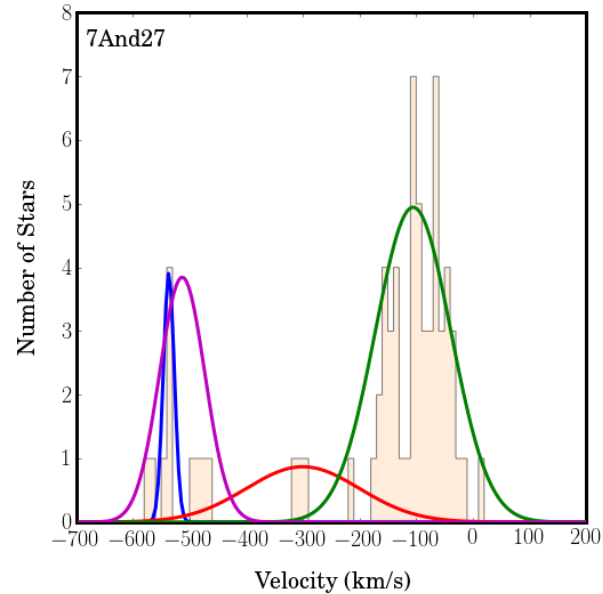

Figure 7: Velocity histogram overlaid with the membership pdf for each of the three stellar populations - shown in magenta for And XXVII (and equivalent to that shown in Figure 3 for this mask), red for M31 and green for the MW. The blue line indicates the pdf for the And XXVII cold peak.

centre of mask 7 And 27 are very close to those for the centre of And XXVII we assign stars in this field to the stellar population for the dSph and assign stars from mask $603 \mathrm{HaS}$ to the stellar population for NW-K1.

The new $\sigma_{v}$ for And XXVII is also larger than many other dwarf galaxies in the Local Group, with only Canis Major, $\sigma_{v}=20.0 \pm 3.0 \mathrm{kms}^{-1}$, the Large Magellanic Cloud, $\sigma_{v}=20.2 \pm 0.5 \mathrm{kms}^{-1}, \mathrm{NGC185}, \sigma_{v}=24.0 \pm 1.0 \mathrm{kms}^{-1}$, the Small Magellanic Cloud, $\sigma_{v}=27.6 \pm 0.5 \mathrm{kms}^{-1}$, NGC205, $\sigma_{v}$ $=35.0 \pm 5.0 \mathrm{kms}^{-1}$ and M32, $\sigma_{v}=92.0 \pm 5.0 \mathrm{kms}^{-1}$, having similar or larger values, McConnachie (2012). Given that And XXVII has a small $\mathrm{r}_{h}$, we take this large $\sigma_{v}$ as a possible indicator that And XXVII is being tidally disrupted.

While constraining the cold peak we notice four stars (with velocities $\sim-500 \mathrm{kms}^{-1}$, see Figure 3 ) that are separate from the main peak and could be affecting our fit of a single Gaussian, causing the large velocity dispersion. Likely to have been categorised as And XXVII candidates stars due to the sample size and breadth of the priors used for fitting the data, we consider the possibility that these stars could be a substructure within And XXVII. Other possible explanations are:

- They are M31 halo stars. With the velocity dispersion, at the distance of this mask, $\sim 99.7 \mathrm{kms}^{-1}$, these stars are only $2 \sigma$ away from the mean of the M31 halo systemic velocity. However, we see that these stars have a greater than $90 \%$ probability of association with And XXVII, so it is unlikely that they are halo stars.

- While these stars may appear to be close to the centre of And XXVII, the imaging data provide only a projected position and they may, in fact, be further away than they seem.

- These stars have been stripped and are no longer in equilibrium with And XXVII.

There is no way to know which of the above is correct. Had the spectra been good enough we might have been able to use $[\alpha / \mathrm{H}]$ abundances to see if the stars were more consistent with And XXVII or M31. However, as they have high probabilities of association with And XXVII, there would seem to be some credence to the possibility that they are stripped stars.

\subsubsection{Velocity Gradients}

Assuming that And XXVII is being tidally disrupted by M31 we look for evidence to support this hypothesis. In the first instance we explore the possibility of a tangential velocity gradient across the centre of And XXVII. Aden et al. (2009) noted that dSphs with such gradients were either undergoing tidal interactions or had an intrinsic rotation. Martin \& Jin (2010) measured a velocity gradient along the major axis of the Hercules dSph and concluded this was indicative of it being pulled apart and transforming into a tidal stream following a close pass of the Milky Way centre.

Using techniques described by Martin \& Jin (2010) and Collins et al. (2016), we amend Equation 3.2 to include a velocity gradient $\left(\frac{d v}{d \chi}\right)$ as shown in 3.10 .

$$
\begin{aligned}
P_{\text {struc }}= & \frac{1}{\sqrt{2 \pi\left(\sigma_{v, \text { struc }}^{2}+\sigma_{\text {sys }}^{2}\right)}} \times \\
& \quad \exp \left[-\frac{1}{2}\left(\frac{\Delta v_{r, i}}{\sqrt{\left.\sigma_{v, \text { struc }}^{2}++\sigma_{\text {sys }}^{2}\right)}}\right)^{2}\right]
\end{aligned}
$$

where $\Delta v_{r, i}\left(\mathrm{kms}^{-1}\right)$ is the velocity difference between the $i^{\text {th }}$ star and a velocity gradient, $\frac{d v}{d \chi}\left(\mathrm{kms}^{-1} \operatorname{arcmins}^{-1}\right)$, acting along the angular distance of the star along an axis, $y_{i}$ (arcmins), with a position angle, $\theta$ (radians, measured from North to East) and is given by:

$\Delta v_{r, i}=v_{r, i}-\frac{d v}{d \chi} y_{i}+\left\langle v_{r}\right\rangle$

We determine $y_{i}$ using the right ascension and declination of the $i^{\text {th }}$ star $\left(\alpha_{i}, \delta_{i}\right)$ and the centre of And XXVII $\left(\alpha_{0}, \delta_{0}\right)$ using:

$y_{i}=X_{i} \sin (\theta)+Y_{i} \cos (\theta)$

where:

$X_{i}=\left(\alpha_{i}-\alpha_{0}\right) \cos \left(\delta_{0}\right) \quad$ and $\quad Y_{i}=\delta_{\mathrm{i}}-\delta_{0}$

We define flat priors for these new terms, i.e. $-150 \leq$ $\frac{d v}{d \chi}\left(\mathrm{kms}^{-1}\right.$ arcmins $\left.^{-1}\right) \leq 150$ and $0 \leq \theta$ (radians) $\leq \pi$ and use the priors from our single Gaussian analysis for the other parameters $\left(v, \sigma_{v}\right.$ and $\left.\eta\right)$ before running the EMCEE algorithm on the confirmed stellar populations on masks 7And27, 603HaS, A27sf1 and A27sf3 as they cover the length of the stream and each includes sufficient And XXVII/NWK1 stars to deliver meaningful results.

Our findings show marginal tangential gradients in all four masks but none are significant or have any effect on the systemic velocities and dispersions recorded in Table 3 . This could, again, be due to the sample size on each mask or could indicate that we are seeing stars, moving at high velocities along a straight line path, that are the debris of a completely unbound system. If this latter is the case, this could also explain the different velocity dispersions we obtain from our analysis of mask 7And27. Simulations by Klimentowski et al. (2007) find that data taken from particular lines of sight can be contaminated by unbound stars in tidal tails, 


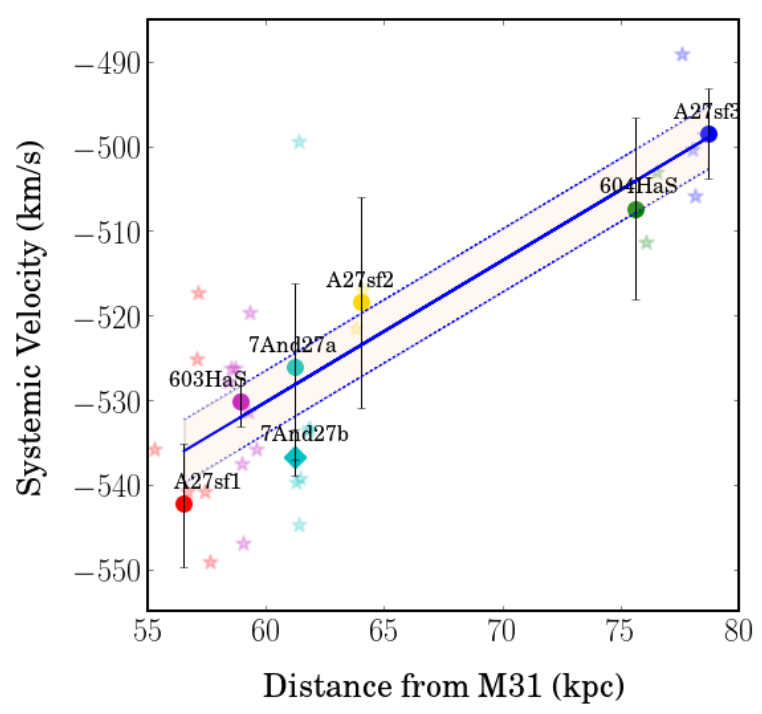

Figure 8: Velocity gradient across all masks with respect to the centre of M31. The values for the velocities and the uncertainties (at $68 \%$ confidence limits) were obtained from the EMCEE algorithm. The mask locations were obtained using the mean value of all the $\alpha \mathrm{s}$ and $\delta \mathrm{s}$ for the stars on each respective mask. The blue line is the best fit line and has a gradient of $-1.7 \pm 0.3 \mathrm{kms}^{-1}$ $\mathrm{kpc}^{-1}$. The shaded area, bounded by dotted lines, indicates the standard deviation $\left( \pm 3.7 \mathrm{kms}^{-1}\right)$ about the best fit line. The star shaped icons represent the And XXVII/NW-K1 confirmed stellar population for each mask, the colour coding denotes the mask on which they were observed.

Next we look to see if there is a velocity gradient across And XXVII and NW-K1. In Figure 8 we present the systemic velocity as a function of the distance of each mask from the centre of M31. Based on line of sight data, this plot shows a distinctive velocity gradient $\left(-1.7 \pm 0.3 \mathrm{kms}^{-1}\right.$ $\mathrm{kpc}^{-1}$ ) that becomes increasingly negative in the direction of M31 - indicative of an infall trajectory. The small scatter of the mask velocities around the best fit line implies that the stars belong to a dynamically cold system.

We compare these findings with those of Mackey et al. (2010), Veljanoski et al. (2013) and Veljanoski et al. (2014) on Globular Clusters (GC) projected onto NWK2. Veljanoski et al. (2014) find a velocity gradient of $1.0 \pm 0.1 \mathrm{kms}^{-1} \mathrm{kpc}^{-1}$ across the GCs, that becomes increasingly negative in the direction of M31.

With both NW-K1 and NW-K2 appearing to have trajectories infalling towards M31 we hypothesise that they cannot be part of the same structure. However, we note that as we only have line of sight velocities, the gradients could be due to the shape of the stream and there could be stronger, undetectable, velocity components in directions compatible with a single structure. Since Komiyama et al. (2018) find both sections of the NW stream lie behind M31 for them both to have infall trajectories towards M31 is difficult to reconcile with a single stellar structure and it is more probable that they are different streams.

\subsection{Metallicities}

We measure the spectroscopic metallicities of the stars in And XXVII /NW-K1 using the CaT lines between $8400 \AA$ -
$8700 \AA$. As the $\mathrm{S} / \mathrm{N}>3$ for only 12 stars, we conclude that using the individual spectra to determine metallicities would not deliver robust results.

In low $\mathrm{S} / \mathrm{N}$ spectra, the CaT lines are hard to distinguish from the noise and are, therefore, unreliable. As we see only a small spread of metallicities on the CMD, we choose to stack the spectra (following the approach of Ibata et al. 2005a, Chapman et al. 2005, 2007 and Collins et al. 2010, 2011) as combining them will likely give a good estimate of the average. We acknowledge that the approach may deliver a more metal-rich result than expected while recognising that the technique provides a good indication of mean values.

We prepare the individual spectra, following the method described by Collins et al. (2013), by correcting for the velocity of the individual star, smoothing the spectrum and normalising the data using a median filter. We weight each spectrum by its $\mathrm{S} / \mathrm{N}$ and interpolate the spectra to a consistent framework before co-adding the fluxes. We simultaneously fit the continuum and the CaT lines to the co-added spectrum (see example shown at Figure 9) to obtain the equivalent widths.

The relationship between equivalent width and $[\mathrm{Fe} / \mathrm{H}]$ of an object is well established (Rutledge et al. 1997, Battaglia et al. 2008, Starkenburg et al. 2010) and has been calibrated for use with all three, or subsets thereof, CaT lines. As not all of our co-added spectra have three well defined CaT lines (i.e. our first line is often contaminated by sky-lines) and as the CMD indicates our stars are metalpoor, we follow the approach described by Starkenburg et al. (2010) and substitute the equivalent widths obtained above into:

$[\mathrm{Fe} / \mathrm{H}]=a+b M+c E W_{(2+3)}+d E W_{(2+3)}^{-1.5}+e E W_{(2+3)} M$

where: $a, b, c, d$ and $e$ are taken from the calibration to the Johnson-Cousins $M_{I}$ values and equal to $-2.78,0.193,0.442$, -0.834 and 0.0017 respectively; and $\mathrm{EW}_{2}$ and $\mathrm{EW}_{3}$ are the the equivalent widths for the CaT lines at $8542 \AA$ and $8662 \AA$ respectively. $\mathrm{EW}_{(2+3)}=\mathrm{EW}_{2}$ and $\mathrm{EW}_{3} \cdot M$ is the absolute magnitude of the star given by:

$M=i-5 \times \log _{10}\left(\mathrm{D}_{\odot}\right)+5$

where: $i$ is the i-magnitude of the star and $D_{\odot}$ is the heliocentric distance for the star, which we assume, in all cases, to be the heliocentric distance for And XXVII. Uncertainties on the metallicity are determined using the uncertainties on the equivalent widths, obtained from the covariance matrix produced by the fitting process, combined in quadrature. Other intrinsic uncertainties in the approach taken are discussed in Starkenburg et al. (2010).

We present our results in Table 4 . We find the metallicity for And XXVII to be $-2.1_{-0.5}^{+0.4}$, while NW-K1 has a mean metallicity of $-1.8 \pm 0.4$. Our values are consistent with previous findings of: $[\mathrm{Fe} / \mathrm{H}]_{\text {phot }}=-1.7 \pm 0.2$, Richardson et al. (2011); $[\mathrm{Fe} / \mathrm{H}]_{\mathrm{spec}}=-2.1 \pm 0.5$, Collins et al. (2013); the metallicity of And XXVII's RR Lyrae stars $=-1.62 \pm 0.23$, Cusano et al. (2017), and with the results from the isochrone analysis.

We plot the metallicity of And XXVII as a function of luminosity (Figure 10). Kirby et al. (2013) established 


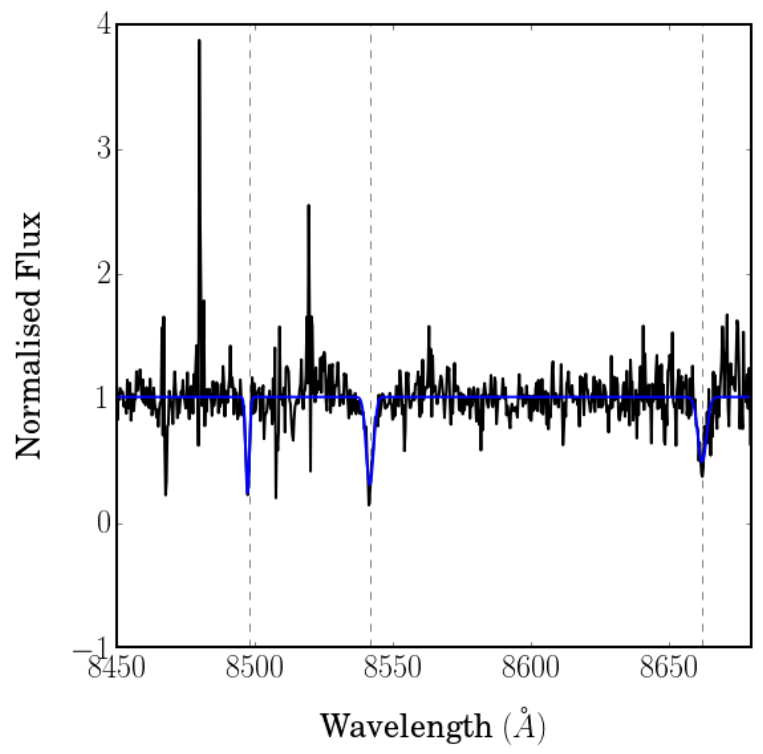

Figure 9: Co-added spectrum from mask $603 \mathrm{HaS}$ overlaid with the best fit curve (solid blue line). This is representative of the results of the spectral analyses conducted for all masks. The dotted lines indicate the position of the CaT.

\begin{tabular}{lc}
\hline Mask & {$[\mathrm{Fe} / \mathrm{H}]_{\mathrm{spec}}$} \\
\hline $\begin{array}{l}\text { And XXVII } \\
7 \text { And27 }\end{array}$ & $-2.1_{-0.5}^{+0.4}$ \\
\hline NW-K1 & $-1.7_{-0.4}^{+0.3}$ \\
A27sf1 & $-1.5 \pm 0.4$ \\
$603 \mathrm{HaS}$ & $-1.6_{-4.0}^{+1.5}$ \\
A27sf2 & $-2.5_{-1.0}^{+0.6}$ \\
$604 \mathrm{HaS}$ & $-1.8 \pm 0.5$ \\
A27sf3 & $-1.4 \pm 0.1$ \\
\hline NW-K1 mean & \\
\hline
\end{tabular}

Table 4: Metallicities obtained from co-added spectra weighted by $S / N$ for stars on each mask.

that the metallicities of MW dSphs decrease with decreasing luminosity in a relationship given by:

$$
\langle[\mathrm{Fe} / \mathrm{H}]\rangle_{\mathrm{dSph}}=(-1.69 \pm 0.06)+(0.29 \pm 0.04) \log \left(\frac{L_{v}}{10^{6} L_{\odot}}\right)
$$

where: $\langle[\mathrm{Fe} / \mathrm{H}]\rangle_{\mathrm{dSph}}$ is the average weighted mean metallicity of the galaxy and $\mathrm{L}_{v}$ is its corresponding luminosity. Collins et al. (2013) showed that the M31 dSphs also conform to this relationship. Our plot shows that And XXVII's metallicity lies within $1-\sigma$ of the best fit line and is consistent with Local Group dwarf galaxies of similar luminosities.

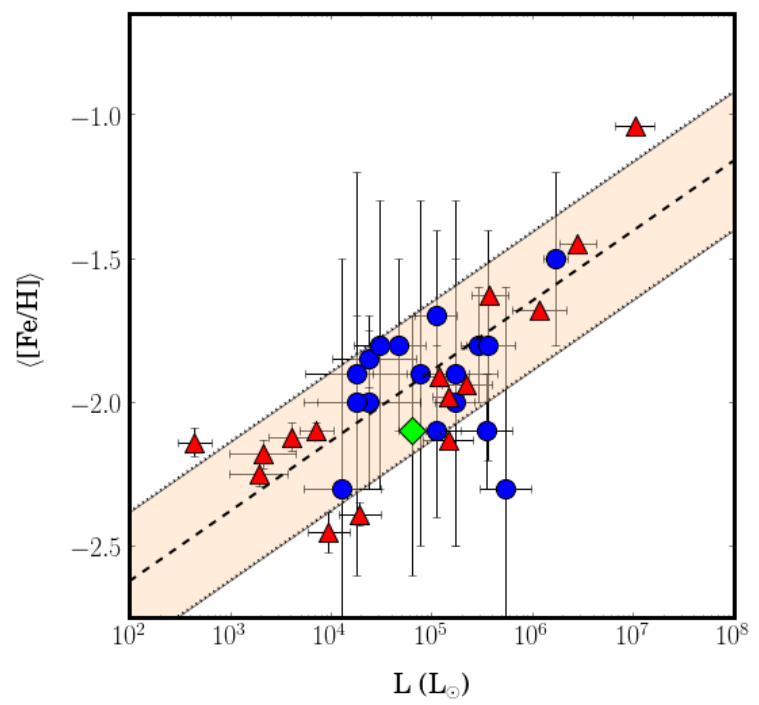

Figure 10: Spectroscopic metallicities as a function of luminosity at the half light radius for Local Group dwarf galaxies with those from M31 shown as blue circles, those from MW shown as red triangles and And XXVII/NW-K1 shown as a green diamond. The dashed line indicates the best fit line, following the approach by Kirby et al. (2013). The shaded area, bounded by dotted lines, indicates the $1 \sigma$ deviation. Data sources: Collins et al. (2013) and Kirby et al. (2013) and Ho et al. (2014).

\section{CONCLUSIONS}

We present our kinematic and spectroscopic analysis of 38 red giant branch stars from 7 fields, including 4 new ones, spanning And XXVII and NW-K1. We have confirmed secure members of the stellar populations belonging to both features defined by strong $(\geq 1 \sigma)$ association with our fiducial isochrone and with the systemic velocity for And XXVII (see Table 5). Our results lead us to conclude:

- The heliocentric distance for And XXVII is $827 \pm 47$ $k p c$. Determined by Richardson et al. (2011) this was later revised by Conn et al. (2012) to $1255_{-474}^{+42} \mathrm{kpc}$ using their Tip of the RGB approach. However, their work also shows the distance posterior distribution for And XXVII has a pronounced second peak, indicative of an heliocentric distance $\sim 800 \mathrm{kpc}$. Given this value is consistent with the findings of Richardson et al. and Cusano et al. (2017) and that our confirmed member stars for And XXVII are probably brighter than assumed by Conn et al. (as can be seen in the CMD plot for mask 7And27 at Figure 4, where the dashed isochrone has been distance corrected to $1255 \mathrm{kpc}$ ) we believe the original heliocentric distance to be the most likely.

- And XXVII is likely to be in the process of being tidally stripped by M31. We measure $\sigma_{v}$ for And XXVII to be $\sim 13$ kms higher than previously reported. This new $\sigma_{v}$ is inconsistent with neighbouring NW-K1 fields and with many other dwarf galaxies in the Local Group. However, it is consistent with expectations of a dwarf galaxy in the throes of a tidal disruption event.

- Given the possibility that And XXVII is tidally disrupting and is not in virial equilibrium, we cannot constrain its mass. However, our kinematic results, especially for NW- 
Andromeda XXVII and the North West Stream

\begin{tabular}{|c|c|c|c|c|c|c|c|}
\hline Mask/star & $\alpha$ & $\delta$ & $i$ & $g$ & $S / N$ & $v_{r}$ & $P_{A 27}$ \\
\hline \multicolumn{8}{|l|}{ And XXVII } \\
\hline \multicolumn{8}{|l|}{ 7And27 } \\
\hline 5 & $00: 37: 18.84$ & $+45: 23: 19.3$ & 22.1 & 23.3 & 2.5 & $-463.2 \pm 4.8$ & 0.65 \\
\hline 6 & $00: 37: 19.75$ & $+45: 23: 51.8$ & 21.4 & 22.8 & 4.7 & $-539.6 \pm 4.0$ & 0.98 \\
\hline 7 & $00: 37: 19.82$ & $+45: 24: 17.8$ & 21.6 & 23.0 & 3.9 & $-476.1 \pm 6.4$ & 0.83 \\
\hline 11 & $00: 37: 19.24$ & $+45: 21: 36.4$ & 21.8 & 23.1 & 3.1 & $-563.1 \pm 4.1$ & 0.97 \\
\hline 19 & $00: 37: 33.79$ & $+45: 25: 18.9$ & 22.4 & 23.6 & 2.2 & $-539.2 \pm 9.6$ & 0.99 \\
\hline 20 & $00: 37: 41.84$ & $+45: 25: 27.9$ & 22.0 & 23.5 & 2.7 & $-544.7 \pm 5.2$ & 0.94 \\
\hline 31 & $00: 37: 36.90$ & $+45: 27: 06.8$ & 21.8 & 23.3 & 3.5 & $-533.3 \pm 5.3$ & 0.96 \\
\hline 32 & $00: 37: 43.68$ & $+45: 27: 11.3$ & 21.3 & 23.0 & 5.3 & $-533.5 \pm 3.2$ & 0.97 \\
\hline 46 & 00:37:7.86 & $+45: 22: 50.5$ & 22.6 & 23.8 & 2.0 & $-579.1 \pm 15.6$ & 0.95 \\
\hline 54 & $00: 37: 21.21$ & $+45: 24: 25.2$ & 23.2 & 24.1 & 1.5 & $-499.5 \pm 11.4$ & 0.95 \\
\hline 55 & 00:37:19.59 & $+45: 24: 37.5$ & 23.2 & 24.2 & 0.8 & $-480.4 \pm 4.0$ & 0.91 \\
\hline \multicolumn{8}{|l|}{ NW-K1 } \\
\hline \multicolumn{8}{|l|}{$\mathrm{A} 27 \mathrm{sf} 1$} \\
\hline 11 & $00: 39: 28.22$ & $+45: 09: 7.8$ & 21.3 & 23.0 & 5.3 & $-540.9 \pm 3.2$ & 0.87 \\
\hline 23 & $00: 40: 12.21$ & $+45: 04: 21.1$ & 22.1 & 23.4 & 2.2 & $-535.8 \pm 7.6$ & 0.97 \\
\hline 31 & $00: 39: 37.65$ & $+45: 8: 52.8$ & 22.8 & 24.0 & 1.3 & $-560.0 \pm 16.5$ & 0.98 \\
\hline 32 & $00: 39: 24.05$ & $+45: 10: 26.6$ & 22.9 & 23.9 & 1.4 & $-571.2 \pm 5.6$ & 0.98 \\
\hline 33 & $00: 39: 18.52$ & $+45: 10: 29.4$ & 22.8 & 24.0 & 1.8 & $-525.1 \pm 12.1$ & 0.92 \\
\hline 35 & $00: 39: 30.70$ & $+45: 11: 07.3$ & 22.7 & 24.0 & 1.6 & $-517.3 \pm 11.0$ & 0.9 \\
\hline 37 & 00:39:22.19 & $+45: 11: 56.9$ & 22.6 & 24.0 & 1.6 & $-540.9 \pm 9.6$ & 0.83 \\
\hline 38 & $00: 39: 25.79$ & $+45: 12: 53.9$ & 22.6 & 23.9 & 1.4 & $-549.1 \pm 16.7$ & 0.93 \\
\hline \multicolumn{8}{|l|}{$603 \mathrm{HaS}$} \\
\hline 10 & 00:39:8.53 & $+45: 15: 46.8$ & 21.2 & 23.0 & 4.6 & $-527.9 \pm 5.0$ & 0.86 \\
\hline 15 & $00: 39: 5.93$ & $+45: 16: 55.3$ & 22.1 & 23.4 & 2.2 & $-526.2 \pm 3.1$ & 0.99 \\
\hline 20 & $00: 39: 25.73$ & $+45: 19: 55.0$ & 22.4 & 23.6 & 1.5 & $-531.5 \pm 4.0$ & 1.0 \\
\hline 32 & $00: 38: 30.15$ & $+45: 18: 18.1$ & 21.7 & 23.1 & 3.0 & $-519.6 \pm 6.0$ & 0.99 \\
\hline 33 & 00:38:46.02 & $+45: 17: 28.4$ & 21.2 & 23.2 & 4.8 & $-537.5 \pm 4.0$ & 0.6 \\
\hline 35 & $00: 38: 38.75$ & $+45: 17: 33.4$ & 21.7 & 23.2 & 3.4 & $-546.9 \pm 8.1$ & 0.98 \\
\hline 38 & 00:38:44.39 & $+45: 15: 36.2$ & 21.7 & 23.3 & 3.6 & $-526.2 \pm 5.2$ & 0.91 \\
\hline 46 & 00:38:32.03 & $+45: 19: 34.1$ & 22.3 & 23.7 & 1.7 & $-535.8 \pm 17.3$ & 0.9 \\
\hline \multicolumn{8}{|l|}{$\mathrm{A} 27 \mathrm{sf} 2$} \\
\hline 33 & $00: 36: 4.85$ & $+45: 31: 17.8$ & 22.6 & 24.0 & 1.5 & $-521.6 \pm 15.5$ & 0.9 \\
\hline 42 & 00:36:42.08 & $+45: 34: 11.6$ & 22.5 & 23.7 & 1.1 & $-517.0 \pm 8.3$ & 1.0 \\
\hline \multicolumn{8}{|l|}{$604 \mathrm{HaS}$} \\
\hline 16 & $00: 31: 44.15$ & $+46: 11: 9.8$ & 21.6 & 23.0 & 2.7 & $-503.1 \pm 9.1$ & 0.99 \\
\hline \multicolumn{8}{|l|}{$\mathrm{A} 27 \mathrm{sf} 3$} \\
\hline 7 & 00:30:33.8 & $+46: 10: 30.1$ & 21.6 & 23.1 & 8.7 & $-489.1 \pm 7.9$ & 0.98 \\
\hline 31 & 00:30:33.67 & $+46: 13: 04.5$ & 22.2 & 23.6 & 2.4 & $-505.8 \pm 7.3$ & 0.93 \\
\hline 32 & $00: 30: 45.72$ & $+46: 13: 25.7$ & 22.3 & 23.7 & 2.0 & $-500.4 \pm 11.3$ & 0.96 \\
\hline 35 & $00: 30: 32.56$ & $+46: 14: 45.8$ & 22.6 & 23.7 & 1.6 & $-498.7 \pm 3.8$ & 0.97 \\
\hline
\end{tabular}

Table 5: Table showing properties of And XXVII and NW-K1 candidate stars. A similar catalogue of all our observed stars is provided electronically with this article. The columns include: (1) Star number; (2) Right Ascension in J2000; (3) Declination in J2000; (4) $i$-band magnitude; (5) $g$-band magnitude; (6) $S / N\left(\AA^{-1}\right)$; (7) line of sight heliocentric velocity, $v\left(\mathrm{kms}^{-1}\right)$; (8) Probability of membership of And XXVII and NW-K1 as defined in Equation 3.7.

K1, may be useful for mapping the orbit of And XXVII and determining its pre-infall halo mass (Preston et al, in prep).

- And XXVII is a plausible candidate to be the progenitor of $N W-K 1$ : We see consistent properties in the stellar populations for And XXVII and NW-K1 both kinematically, with systemic velocities of $-542.3_{-7.4}^{+7.1} \leq \mathrm{v} / \mathrm{kms}^{-1} \leq-498.6 \pm 5.3$ and spectroscopically with $-1.5 \leq[\mathrm{Fe} / \mathrm{H}] \leq-2.5$. In addition we find a velocity gradient consistent with an infall trajectory towards M31 across And XXVII and NW-K1. Taken together, this indicates that And XXVII and NW-K1 are, potentially, elements of a single feature of which And XXVII may very possibly be the progenitor. 
- The NW Stream may not be not a single structure: The velocity gradients from both $\mathrm{NW}-\mathrm{K} 1$ and NW-K2 are indicative of infall trajectories towards M31. As both streams may lie behind M31, this is difficult to reconcile with a single trajectory looping around M31. Therefore, we conclude that it more likely that they are different streams, with NW-K1 associated with And XXVII and NW-K2 not.

\section{ACKNOWLEDGEMENTS}

JP wishes to thank Marla Geha for her useful discussions and advice on this work. We also thank the anonymous referee who's insightful questions and comments enhanced this paper.

This work used the community-developed software packages: Matplotlib (Hunter 2007), NumPy (van der Walt et al. 2011) and Astropy (Astropy Collaboration et al. 2013, 2018).

Most of the data presented herein were obtained at the W.M. Keck Observatory, which is operated as a scientific partnership among the California Institute of Technology, the University of California and the National Aeronautics and Space Administration. The Observatory was made possible by the generous financial support of the W.M. Keck Foundation.

Data were also used from observations obtained with MegaPrime/MegaCam, a joint project of CFHT and CEA/DAPNIA, at the Canada-France-Hawaii Telescope which is operated by the National Research Council of Canada, the Institut National des Sciences de l'Univers of the Centre National de la Recherche Scientifique of France, and the University of Hawaii.

The authors wish to recognise and acknowledge the very significant cultural role and reverence that the summit of Mauna Kea has always had within the indigenous Hawaiian community.

\section{REFERENCES}

Abbott T., Aldering G., Annis J., Barlow M., Bebek C., Bigelow B., 2005, arXiv:astro-ph/0510346v1

Aden D., Wilkinson M. I., Read J. I., Feltzing S., Koch A., Gilmore G. F., Grebel E. K., Lundstrom I., 2009, The Astrophysical Journal Letters, 706, L150

Ahn C. P., Alexandroff R., Prieto C. A., Anders F., Anderson S. F., 2014, The Astrophysical Journal Supplement Series, 211,17

Akaike H., 1970, Annals of the Institute of Statistical Mathematics, 22,203

Astropy Collaboration et al. 2013, Astronomy and Astrophysics, 558, A33

Astropy Collaboration et al. 2018, The Astronomical Journal, 156,123

Bate N. F., et al., 2014, Monthly Notices of the Royal Astronomical Society, 437, 3362

Battaglia G., Irwin M., Tolstoy E., Hill V., Helmi A., Letarte B., Jablonka P., 2008, Monthly Notices of the Royal Astronomical Society, 383, 183

Belokurov V., et al., 2007, The Astrophysical Journal, 658, 337

Belokurov V., et al., 2014, Monthly Notices of the Royal Astronomical Society, 437, 116
Bernard E. J., et al., 2016, Monthly Notices of the Royal Astronomical Society, 463, 1759

Burnham K. P., Anderson D. R., 2004, Sociological Methods and Research, 33, 261

Carlberg R. G., 2012, The Astrophysical Journal, 748, 20

Carlberg R. G., et al., 2011, The Astrophysical Journal, 731, 124

Carlin J. L., Sand D. J., 2018, arXiv:1805.11624v2

Chapman S. C., Ibata R., Lewis G. F., Ferguson A. M. N., Irwin M., McConnachie A., Tanvir N., 2005, The Astrophysical Journal, 632, L87

Chapman S. C., Ibata R., Lewis G. F., Ferguson A. M. N., Irwin M., McConnachie A., Tanvir N., 2006, The Astrophysical Journal, 653, 255

Chapman S. C., et al., 2007, The Astrophysical Journal, 662, L79?L82

Collins M. L. M., et al., 2010, Monthly Notices of the Royal Astronomical Society, 407, 2411

Collins M. L. M., et al., 2011, Monthly Notices of the Royal Astronomical Society, 413, 1548

Collins M. L. M., et al., 2013, The Astrophysical Journal, 768, 172

Collins M. L. M., Tollerud E. J., Sand D. J., Bonaca A., Willman B., Strader J., 2016, arXiv:1608.05710v1

Conn A. R., et al., 2012, The Astrophysical Journal, 758, 11

Cusano F., et al., 2017, arXiv:1710.10286v1

Dotter A., Chaboyer B., Jevremovic D., Kostov V., Baron E., Ferguson J. W., 2008, The Astrophysical Journal Supplement Series, 178, 89

Erkal D., Belokurov V., Bovy J., Sanders J. L., 2016, Monthly Notices of the Royal Astronomical Society, 463, 102

Erkal D., et al., 2018, arXiv:1812.08192

Fardal M. A., et al., 2012, Monthly Notices of the Royal Astronomical Society, 423, 3134

Fardal M. A., et al., 2013, Monthly Notices of the Royal Astronomical Society, 434, 2779

Ferguson A. M. N., Mackey A. D., 2016, Astrophysics and Space Science Library, 420, 191

Ferguson A. M. N., Irwin M. J., Ibata R. A., F. G. L., Tanvir N. R., 2002, The Astronomical Journal, 124, 1452

Ferguson A. M. N., Johnson R. A., Faria D. C., Irwin M. J., Ibata R. A., Johnston K. V., Lewis G. F., Tanvir N. R., 2005, The Astrophysical Journal, 622, L109

Ferguson A., Irwin M., Chapman S., Ibata R., Lewis G., Tanvir N., 2006, arXiv:astro-ph/0601121v1

Foreman-Mackey D., Hogg D. W., Lang D., Goodman J., 2013, Publications of the Astronomical Society of the Pacific, 125, 306

Foreman-Mackey D., et al., 2016, corner.py v2.0.0

Frenck C. S., White S. D. M., 2012, Annalen der Physik, 524, 507

Gilbert K. M., et al., 2009, The Astrophysical Journal, 705, 1275

Goodman J., Weare J., 2010, Communications in Applied Mathematics and Computational Science, 5, 65

Grillmair C. J., 2006, The Astrophysical Journal, 645, L37

Grillmair C. J., 2009, The Astrophysical Journal, 693, 1118

Grillmair C. J., Carlin J. L., 2016, arXiv:1603.08936v1

Guhathakurta P., et al., 2006, The Astronomical Journal, 131, 2497

Ho N., Geha M., Tollerud E. J., Zinn R., Guhathakurta P., Vargas L. C., 2014, The Astrophysical Journal, 798, 77

Hogg D. W., Foreman-Mackey D., 2017, arXiv:1710.06068v1

Hunter J. D., 2007, Computing in Science and Engineering, 9, 90

Ibata R. A., Gilmore G., Irwin M. J., 1994, Nature, 370, 194

Ibata R., Irwin M., Lewis G., N. A. F. M., Tanvir N., 2001, Nature, 412,49

Ibata R. A., Lewis G. F., Irwin M. J., Quinn T., 2002, Monthly Notices of the Royal Astronomical Society, 332, 915

Ibata R., Chapman S., Ferguson A. M. N., Irwin M., Lewis G., 
McConnachie A., 2004, Monthly Notices of the Royal Astronomical Society, 351, 117

Ibata R., Chapman S., Ferguson A. M. N., Lewis G., Irwin M., Tanvir N., 2005a, American Astronomical Society, 37, 461

Ibata R., Chapman S., Ferguson A. M. N., Lewis G., Irwin M., Tanvir N., 2005b, The Astrophysical Journal, 634, 287

Ibata R., Sollima A., Nipoti C., Bellazzini M., Chapman S. C., Dalessandro E., 2011, The Astrophysical Journal, 738, 186

Ibata R. A., et al., 2014, Astrophysical Journal, 780, 128

Ibata R. A., Malhan K., Martin N. F., 2019, The Astrophysical Journal, 872, 152

Irwin M., Lewis J., 2001, New Astronomy Reviews, 45, 105

Johnston K. V., Spergel D. N., Hernquist L., 1995, The Astrophysical Journal, 451, 598

Kalirai J. S., et al., 2010, The Astrophysical Journal, 711, 671

Kirby E. N., Cohen J. G., Guhathakurta P., Cheng L., Bullock J. S., Gallazzi A., 2013, The Astrophysical Journal, 779, 102

Kirihara T., Miki Y., Mori M., 2017, arXiv:1705.03030v1

Klimentowski J., Lokas E. L., Kazantzidis S., Prada F., Mayer L., Mamon G. A., 2007, Monthly Notices of the Royal Astronomical Society, 378, 353

Komiyama Y., et al., 2018, The Astrophysical Journal, 853, 29

Koposov S. E., Rix H.-W., Hogg D. W., 2010, The Astrophysical Journal, 712, 260

Koposov S. E., et al., 2018, arXiv:1812.08172

Lewis G. F., Ibata R. A., Chapman S. C., Ferguson A. M. N., McConnachie A. W., Irwin M. J., Tanvir N., 2004, The Astronomical Society of Australia, 21, 203

Lewis G. F., et al., 2013, The Astrophysical Journal, 763, 4

Lux H., Read J. I., Lake G., Johnston K. V., 2013, Monthly Notices of the Royal Astronomical Society, 436, 2386

Mackey A. D., et al., 2010, The Astrophysical Journal Letters, $717, \mathrm{~L} 11$

Mackey A. D., et al., 2013, Monthly Notices of the Royal Astronomical Society, 429, 281

Magnier E. A., Cuillandre J. C., 2004, The Publications of the Astronomical Society of the Pacific, 116, 449

Malhan K., Ibata R. A., 2018, arXiv:1807.05994v1

Martin N. F., Jin S., 2010, The Astrophysical Journal, 721, 1333

Martin N. F., et al., 2014a, The Astrophysical Journal, 787, 19

Martin N. F., et al., 2014b, The Astrophysical Journal Letters, 793, L14

Martin N. F., et al., 2016, arXiv:1610.01158v1

Mateo M., 1998, Annual Review of Astronomy and Astrophysics, 36,435

McConnachie A. W., 2012, The Astronomical Journal, 144, 4

McConnachie A. W., Irwin M. J., Ibata R. A., Ferguson A. M. N., Lewis G. F., Tanvir N., 2003, Monthly Notices of the Royal Astronomical Society, 343, 1335

McConnachie A. W., Irwin M. J., Lewis G. F., Ibata R. A., Chapman S. C., Ferguson A. M. N., Tanvir N. R., 2004, Monthly Notices of the Royal Astronomical Society, 351, L94

McConnachie A., et al., 2009, Nature, 461, 66

McConnachie A. W., et al., 2018, The Astrophysical Journal, 868, 55

Newberg H. J., Yanny B., Willet B. A., 2009, The Astrophysical Journal, 700, L61

Press W. H., Schechter P., 1974, The Astrophysical Journal, 187, 425

Richardson J. C., et al., 2011, Astrophysical Journal, 732, 76

Rutledge G. A., Hesser J. E., Stetson P. B., 1997, Astronomical Society of the Pacific, 109, 907

Shipp N., et al., 2018, arXiv:1801.03097v1

Simon D. J., Geha M., 2007, The Astrophysical Journal, 670, 313

Springel V., Frenck C. S., White S. D. M., 2006, Nature, 440, 1137

Starkenburg E., et al., 2010, Astronomy and Astrophysics, 513, id.A34, 15 pp.
The Gaia Collaboration 2016, arXiv:1609.04153v1

Tollerud E. J., et al., 2012, The Astrophysical Journal, 752

Veljanoski J., et al., 2013, The Astrophysical Journal Letters, 768, L33

Veljanoski J., et al., 2014, Monthly Notices of the Royal Astronomical Society, 442, 2929

Zucker D. B., et al., 2004, The Astrophysical Journal, 612, L117

van der Walt S., Colbert C. S., Varoquaux G., 2011, Computing in Science and Engineering, 13, 22 